\title{
Intraductal papillary mucinous neoplasm of the pancreas (IPMN): clinico-pathological correlations and surgical indications
}

Gian Luca Baiocchi ${ }^{1 *}$, Nazario Portolani ${ }^{1}$, Guido Missale ${ }^{2}$, Carla Baronchelli ${ }^{3}$, Federico Gheza ${ }^{1}$, Massimiliano Cantù ${ }^{1}$, Luigi Grazioli ${ }^{4}$, Stefano M Giulini ${ }^{1}$

\begin{abstract}
Background: Intraductal papillary mucinous neoplasms (IPMNs) are increasingly recognized entities, whose management remains sometimes controversial, due to the high rate of benign lesions and on the other side to the good survival after resection of malignant ones.

Methods: Retrospective analysis of a prospectively collected Western series of IPMN.

Results: Forty cases of IPMN were analysed (1992-2007). Most patients were symptomatic (72.5\%); cholangio-MRI had the best diagnostic accuracy both for the tumour nature (83.3\%) and for the presence of malignancy (57.1\%). ERCP was done in 8 cases (20\%), and the results were poor. Thirteen patients were treated by pancreatic resection and 27 were maintained in follow-up. Total pancreatectomy was performed in $46 \%$ of the cases; in situ and invasive carcinoma were recognized in $15.4 \%$ and $38.4 \%$ of the cases, respectively. The mean follow-up was 42 months (range 12-72). One only patients with nodal metastases died 16 months after the operation for disease progression, while $91.6 \%$ of the operated patients are disease free. Out of the 27 not resected patients, 2 out of 4 presenting a lesion at high risk for malignancy died, while the remaining are in good conditions and disease free, with a mean follow-up of 31 months.

Conclusion: Therapeutic indication for IPMNs is mainly based upon radiological evaluation of the risk of malignancy. While the main duct tumours should be resected, preserving whenever possible a portion of the gland, the secondary ducts tumours may be maintained under observation, in absence of radiological elements of suspicion such as size larger than $3 \mathrm{~cm}$, or a wall greater than $3 \mathrm{~mm}$ or nodules or papillae in the context of the cyst.
\end{abstract}

\section{Background}

In the group of cystic neoplasms of the pancreas, the intraductal papillary mucinous tumor (IPMN) represents a recently characterized entity; this denomination was introduced in 1996 [1], and comprises a group of lesions that differ from cystic mucinous neoplasms because of a direct communication with the Wirsung duct and the absence of ovarian-type stroma [2]; it is characterized by a papillary growth of the ductal epithelium with rich mucin production and cystic expansion of the interested duct (Fig. 1).

\footnotetext{
* Correspondence: baiocchi@med.unibs.it

${ }^{1}$ Department of Medical and Surgical Sciences, Surgical Clinic, Brescia University, P.le Spedali Civili, 1, 25123 Brescia, Italy
}

IPMN was firstly described in 1982 [3]; a sharp increase in the frequency of such observations in the following years sets the doubt of a possible new environmental stimulus or a genetic mutation: the hypothesis that before 1980 the IMPNs were simply otherwise classified clashes with the lack of findings of similar tumours in some retrospective revisions [4]. Natural history of this tumour is different from ductal adenocarcinoma: in $90-100 \%$ of the cases it is resectable, with survival reaching $80-90 \%$ for in situ carcinoma, $50-70 \%$ for invasive carcinoma and 40-50\% when nodal metastases are already present [5]. Some preoperative indicators of malignancy were proposed, and their accuracy is actually under prospective evaluation [2].
C Biomed Central

(C) 2010 Baiocchi et al; licensee BioMed Central Ltd. This is an Open Access article distributed under the terms of the Creative Commons Attribution License (http://creativecommons.org/licenses/by/2.0), which permits unrestricted use, distribution, and reproduction in any medium, provided the original work is properly cited. 


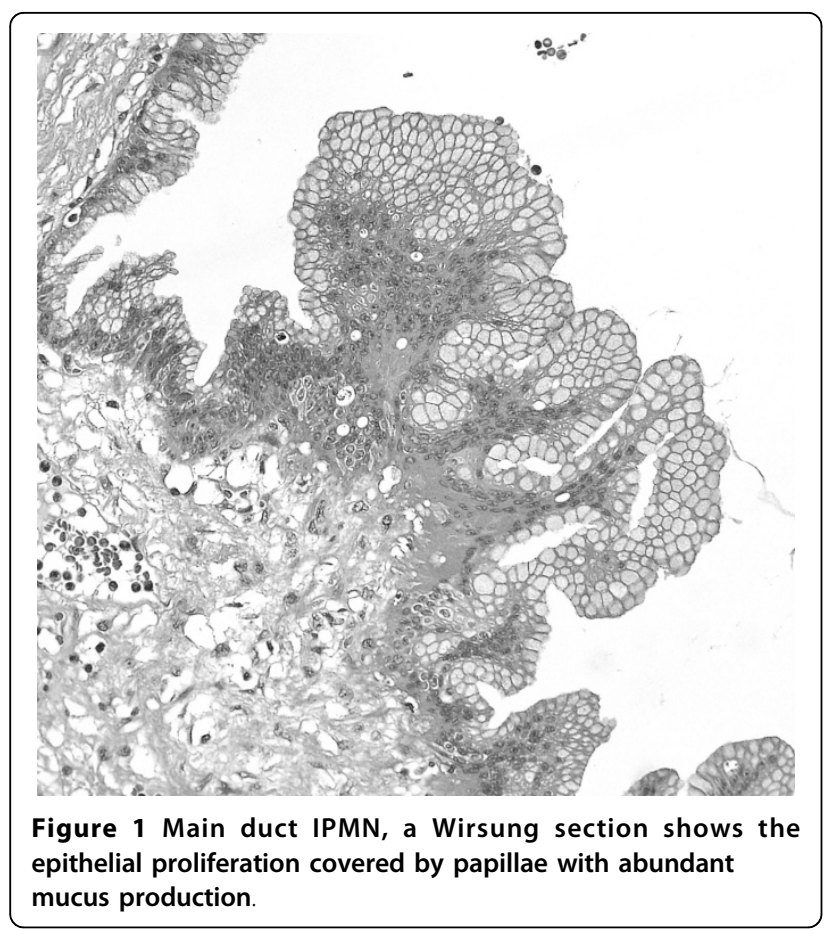

In this paper we present the series of cystic intraductal tumours observed by the Surgical Clinic of the Brescia University, with the aim to underline the clinical problems they set, related both to the therapeutic indication and to the extension of surgical demolition, and to compare the results with those previously reported in Literature.

\section{Methods}

All cases with a definite diagnosis of IPMN (i.e., a cystic neoplasm with a demonstrated communication with or a direct involvement of the Wirsung duct) were taken into consideration. In the period 1992 to 2007, 276 patients affected by pancreas neoplasm have been submitted to surgical exploration, and 186 have been resected, 13 of them with a definitive pathological diagnosis of intraductal papillary mucinous tumor; starting from 2004, on the basis of the literature indications, the cases of IPMN of the secondary ducts of small size have been maintained in follow-up (23 patients); further 4 cases of IPMN suspected for malignancy were not submitted to surgery for an elevated surgical risk (advanced age, cardio-respiratory and liver morbidity); so, the present paper analyzes in total 40 patients with IPMN, 13 treated by surgery and 27 submitted to follow-up.

We considered and recorded as possible risk factors for malignancy smoke, alcoholism, carcinogens exposure, diabetes mellitus, biliary lithiasis, chronic pancreatitis, neoplasms of other organs [5]. CEA and CA 19.9 were recorded in all the patients. Imaging has been analyzed to verify the diagnostic accuracy both for the diagnosis of nature and for the diagnosis of malignancy. In the group of patients submitted to invasive investigations (ERCP with brushing and/or biopsy-aspiration of the cystic content) we considered indications, complications and the influence of the results upon the final therapeutic decision. At the conclusion of the diagnostic workup, IPMNs were subdivided into main duct tumours (MDTs) and branch duct tumours (BDTs). In accordance with the Literature, an high risk for malignancy was hypothesized for all MDT and for BDT larger than $3 \mathrm{~cm}$, or with a wall greater than $3 \mathrm{~mm}$ or with nodules of papillae in the context of the cyst [2]. For diagnostic considerations, those criteria were employed also for the cases observed before the publication of the International Consensus Guidelines in 2006; previous cases were retrospectively re-evaluated.

In patients submitted to surgical intervention, the extension of the resection has always been driven by the preoperative evaluation of the dilated Wirsung duct and by the intraoperative histological examination of the pancreatic cut surface in partial pancreatectomy: in case of presence of IPMN in the cut surface, even if without dysplasia, we extended the resection until a completely negative cut section. In the final histopathological examination the WHO classification was followed [6], dividing the cases into 4 groups: IPMAdenoma (dysplasia of low degree), IPMN border-line (dysplasia of moderate degree), in situ IPMCarcinoma and infiltrating IPMCarcinoma. For the 27 patients not submitted to pancreatic resection, the distinction of benign from malignant lesions has been based on the evolution at follow-up, as already proposed [2].

The mean follow-up was 42 months (range 12-72), 47 months (range 15-72) for the operated patients and 31 months (range 12-51) for the observed ones. The follow-up protocol for the operated patients foresees controls at $3,6,12,18,24$ months and subsequently every 12 months up to 5 years. Such controls included clinical examination, tumour markers, abdominal ultrasound or CT or cholangio-MRI, according to the clinical suspicion (in absence of any suspect of recurrence, CT every year for patients with carcinoma and cholangio-MRI every year for patients with benign neoplasm were performed). In the not resected patients, instead, the follow-up was almost entirely founded on the cholangio-MRI, to reduce exposure of the patient to radiations.

\section{Results}

The relative incidence of the IPMNs in the pancreatic surgical series of the Brescia University Surgical Clinic clearly increased from the period 1992-2003 to 2004-2007: 3/122 (2.4\%) versus $10 / 64$ resections (15.6\%). The 40 patients affected by IPMN were 21 women and 19 males (Table 1 ). 
Table 1 Clinical characteristics of 40 patients with intraductal papillary mucinous neoplasm (IPMN)

\begin{tabular}{|c|c|c|}
\hline & n. pts. & $\%$ \\
\hline \multicolumn{3}{|l|}{ Patients } \\
\hline Middle age (range) & \multicolumn{2}{|c|}{68.3 years $(36-83)$} \\
\hline Male (mean age) & \multicolumn{2}{|c|}{21 (73 years) } \\
\hline Female (mean age) & \multicolumn{2}{|c|}{$19(65.9)$} \\
\hline \multicolumn{3}{|l|}{ Presence of risk factors } \\
\hline Biliary lithiasis & 7 & 17.5 \\
\hline Other tumours & 5 & 12.5 \\
\hline Symptomatic at diagnosis & 29 & 72.5 \\
\hline Acute Pancreatitis & 18 & 45.0 \\
\hline Abdominal pain & 4 & 10.0 \\
\hline Diabetes & 1 & 2.5 \\
\hline Diarrhea & 1 & 2.5 \\
\hline Jaundice & 1 & 2.5 \\
\hline Anorexia & 1 & 2.5 \\
\hline \multicolumn{3}{|l|}{ Tumor markers } \\
\hline Raised Ca 19.9 & 3 & 7.5 \\
\hline Raised CEA & 0 & \\
\hline
\end{tabular}

The majority of them were symptomatic (29/40, 72.5\%); such percentage was slightly different in resected $(61.5 \%)$ and not resected patients $(77.7 \%), \mathrm{p}=0.19$. The most frequent symptoms were acute pancreatitis (18 cases, $62 \%$ of the symptoms and $45 \%$ of all the patients with IPMN) and abdominal pain (4 cases, $13.7 \%$ of the symptoms). In 11 cases $(27.5 \%)$ the IPMN was incidentally discovered during ultrasonographic investigation performed for follow-up of other neoplasms (5 cases) or for different reasons (6 cases). An increase of CA 19.9 was documented in 3 patients, while CEA always resulted normal.

Table 2 reports data related to the diagnostic course. In most cases the first examination setting the suspicion of IPMN has been the ultrasound (24 cases, $60 \%$ ), after which CT or MRI were done in all the patients, the diagnostic accuracy of cholangio-MRI being clearly superior (83\%), given its ability to show the communication of the cyst with the Wirsung duct. Despite the above examinations substantially brought elements adequate to set the diagnosis of IPMN in 37/40 patients, in

Table 2 Diagnostic accuracy of imaging techniques employed in 40 patients with IPMN

\begin{tabular}{|c|c|c|c|c|c|c|}
\hline & \multicolumn{2}{|c|}{$\begin{array}{l}\text { ultrasound } \\
\text { (26 pts.) }\end{array}$} & \multicolumn{2}{|c|}{$\begin{array}{c}C T \\
\text { (31 pts.) }\end{array}$} & \multicolumn{2}{|c|}{$\begin{array}{c}\text { MR } \\
\text { (24 pts.) }\end{array}$} \\
\hline & $\mathbf{n}$ & $\%$ & $\mathbf{n}$ & $\%$ & n & $\%$ \\
\hline Correct diagnosis of IPMN & 4 & 15.4 & 11 & 35.4 & 20 & 83.3 \\
\hline Correct diagnosis of malignancy ${ }^{\circ}$ & $1 / 3$ & 33.3 & $2 / 4$ & 50.0 & $4 / 7$ & 57.1 \\
\hline Correct diagnosis of extension§ & 1 & 25.0 & $9 / 11$ & 81.8 & 22 & 100 \\
\hline
\end{tabular}

- Reference parameter was final histological diagnosis of malignant IPMN §Reference parameter was macroscopic pathology for resected patients and MRI for the others many cases the radiologist required a diagnostic confirmation by ERCP. Nevertheless, ERCP was done only in 8 patients (20\%), and the results were poor, because a correct spatial definition of the Wirsung duct involvement was obtained only in 4 cases (50\%), and the pancreatic brushing was possible only in 5 cases $(62.5 \%)$, with negative results in all but one (resulting doubtful); in $50 \%$ of the cases ERCP was followed by pancreatitis that lasted for various weeks, making it necessary to delay surgical intervention in 2 patients and making more difficult the pancreatic resection in one. In 3 cases we proceeded to aspiration of the cystic content by echo-endoscopy for biochemical and cytological examination, always with negative result. In 7 cases a PET scan was also performed, with 2 positive and 5 negative results [7].

At the completion of the pre-operative study, on the basis of the previously reported criteria [2], malignancy could be suspected in $17 / 40$ patients $(42.5 \%)$ from the morphological characteristics (14 cases), brushing cytology ( 1 case), CA 19.9 rise (3 cases) and indicative symptoms as jaundice and weight loss ( 2 cases). Thirteen out of these 17 patients were operated on and 4 were not, because of high surgical risk. Performed interventions are reported in Table 3. In 2 cases the positive intraoperative frozen section examination of the margin imposed to extend the resection up to a total pancreatectomy. A lymphadenectomy was associated to the pancreatic resection in 7 patients, removing the nodes anterior and posterior to the pancreas head, those of celiac artery, hepatic artery, common bile duct and those of the root of the superior mesenteric vein. It was never necessary to associate vascular and visceral resections.

In all the resected cases the conclusive diagnosis has been IPMN. Ten cases have been classified as main duct tumour (MDT) and 3 as branch duct tumour (BDT). This corresponded to the preoperative classification based on the imaging in all the cases. Location and size are reported in Table 3. If we consider benign the adenoma IPMNs and the border-line IPMNs, and malignant those with carcinoma in situ or infiltrating, 7 resected lesions were malignant $(53,8 \%)$. Based upon the reported morphological criteria [2], and the final assessment of the radiologist, when clearly expressed, the preoperative diagnostic accuracy in differentiating benign from malignant IPMN was $69.2 \%$ (9/13 patients). On the basis of the definitive histological examination, in all the patients the resection has been considered $\mathrm{R} 0$. In no case a positive margin was found at the final pathology. One only patient had metastatic nodes.

We complain the death at 62th post-operative day of the patient, already described, submitted to total pancreatectomy for a main duct IPMN, after having 
Table 3 Surgical and pathological characteristics of 13 patients with resected intraductal papillary mucinous neoplasm (IPMN)

\begin{tabular}{|c|c|c|}
\hline & $\mathrm{N}$. & $\%$ \\
\hline \multicolumn{3}{|l|}{ Therapy } \\
\hline Resection & 13 & 32.5 \\
\hline Mean age (range) & \multicolumn{2}{|c|}{$68.8(57-81)$} \\
\hline Total Pancreatectomy & 6 & 46.1 \\
\hline Left Pancreatectomy & 5 & 38.4 \\
\hline Duodeno-pancreatectomy & 2 & 15.4 \\
\hline Lymphadenectomy & $7^{*}$ & 53.8 \\
\hline \multicolumn{3}{|l|}{ Pathology } \\
\hline MDT & 10 & 76.9 \\
\hline BDT & 3 & 23.1 \\
\hline \multicolumn{3}{|l|}{ Location } \\
\hline Head & 3 & 23.1 \\
\hline Body-Tail & 5 & 38.4 \\
\hline Whole Wirsung & 5 & 38.4 \\
\hline \multicolumn{3}{|l|}{ WHO classification } \\
\hline Adenoma & 4 & 30.7 \\
\hline Borderline & 2 & 15.4 \\
\hline In situ carcinoma & 2 & 15.4 \\
\hline Infiltrating carcinoma & 5 & 38.4 \\
\hline Middle diameter (range) & \multicolumn{2}{|c|}{$3.92 \mathrm{~cm}(1.8-6 \mathrm{~cm})$} \\
\hline Nodal metastases & $1 / 7$ & 14.3 \\
\hline \multicolumn{3}{|l|}{ Postoperative } \\
\hline Mortality & 1 & 7.7 \\
\hline Morbidity & 4 & 30.7 \\
\hline
\end{tabular}

*112 nodes were retrieved in total (mean 16/patient)

experienced acute pancreatitis post ERCP and with a post-operative course complicated by delayed gastric emptying and respiratory sepsis. The overall morbidity of the series $(30.7 \%)$ includes 2 low-flow fistulas, conservatively treated, a gastrojejunostomy hemorrhage, endoscopically treated, and a pulmonary embolism. Among minor complications, 3 pleural effusions, 1 wound infection and 2 troubles of the glycemic control were recorded. Eleven out of the 12 patients who survived after the resection are alive and disease-free. The patient presenting nodal metastases had a relapse after 12 months and deceased 16 months after the operation. Out of the 27 not resected patients, 2 out of 4 presenting a lesion at high risk for malignancy died, the first 6 months after the diagnosis for disease progression, the second at 16 months for decompensation of a pre-existing Child $\mathrm{C}$ liver cirrhosis. The remaining 25 patients, including the 2 remaining whose lesions presented aspects indicative of potential malignancy, are in good conditions and disease free, with a mean follow-up of 31 months.

\section{Discussion}

Malignant IPMN represents a good surgical indication, as Literature survival data report [8-11], and our series confirms. Nevertheless, controversies remain, relative to the diagnostic protocol to be adopted, to the surgical indication in the IPMNs at low risk of malignancy, and finally to the extension of pancreatic resection.

The first problem is the identification of the tumour. IPMNs are symptomatic in most cases, with acute pancreatitis due to duct obstruction from mucus or from the papillary proliferations. In the present series, less than half of the patients had an episode of acute pancreatitis; among these, only $27.7 \%$ had a history of biliary stones and $5.5 \%$ of alcoholism. Only in 1 case imaging demonstrated a severe pancreatitis. Thus, the typical presentation has been a recurrent acute pancreatitis, without evident cause, of low or moderate severity, but with a log-standing asymptomatic hyperlipasemia. Such set of symptoms must lead to suspect the existence of an intraductal cause, that can be confirmed by further diagnostic steps, such as cholangio-MRI. The remaining half of the patients with IPMN are asymptomatic, and the tumour is discovered incidentally, often during abdominal ultrasound performed for other reasons [12]. Also in these cases the colangio-MRI has elective indication to demonstrate the intraductal nature of the lesion, eventually communicating with the Wirsung duct.

Cholangio-MRI is confirmed from our data to be superior to CT scan in the study of IPMN patients (Table 2). It doesn't emerge from our experience any substantial advantage from ERCP. The only potential added value of such an invasive procedure, the Wirsung duct brushing, finds heavy limits in its extremely low sensibility. In our experience, in half cases the contrast medium wasn't able to depict the whole cystic or polycystic lesion shown by cholangio-MRI, because of the density of its mucous content. Furthermore, ERCP may harbour in these patients an increased risk of complications (e. g. acute pancreatitis), which can heavily interfere with the eventual surgical option. Fifty per cent of the IPMN patients submitted to ERCP developed a iatrogenic pancreatitis in our experience, percentage much higher than ERCP for other diseases. One of these patients had a postoperative course dramatically complicated, concluded by death in $62^{\text {th }}$ day. Surprisingly, this idea was not previously underlined in the Literature. There is no evidence that further invasive diagnostic procedures, such as wirsungscopy, echo-endoscopy with biopsy or fine needle sample and percutaneous biopsy, may warrant a substantial improvement in diagnostic accuracy $[13,14]$. Starting from 2006 , we planned a prospective study on the use of 18-FDG-PET to differentiate benign from malignant lesions [7]. Such technique is able to 
demonstrate some increased metabolic signal in nodules or solid papillae lying in the context of the cystic wall [15]. The results gathered from our first 28 patients demonstrated an improvement in specificity compared to cholangio-MRI alone (specificity $43 \%$ with the MRI, $100 \%$ with MRI plus PET), nevertheless such data are in progress and they are not extensively introduced in this paper.

So, the diagnosis of IPMN is usually based upon the imaging (CT/cholangio-MRI) demonstrating a pancreatic cystic mass, involving a dilated main duct, eventually associated to some filling defects (MDT, principal duct), or a normal Wirsung duct communicating with the cyst lesion (BDT, secondary ducts).

Many papers analysed the predictive factors for malignancy in IPMN [16-21]. In a recent Japanese retrospective study [22], 17 clinical, radiological and pathological parameters were considered in 64 patients operated on

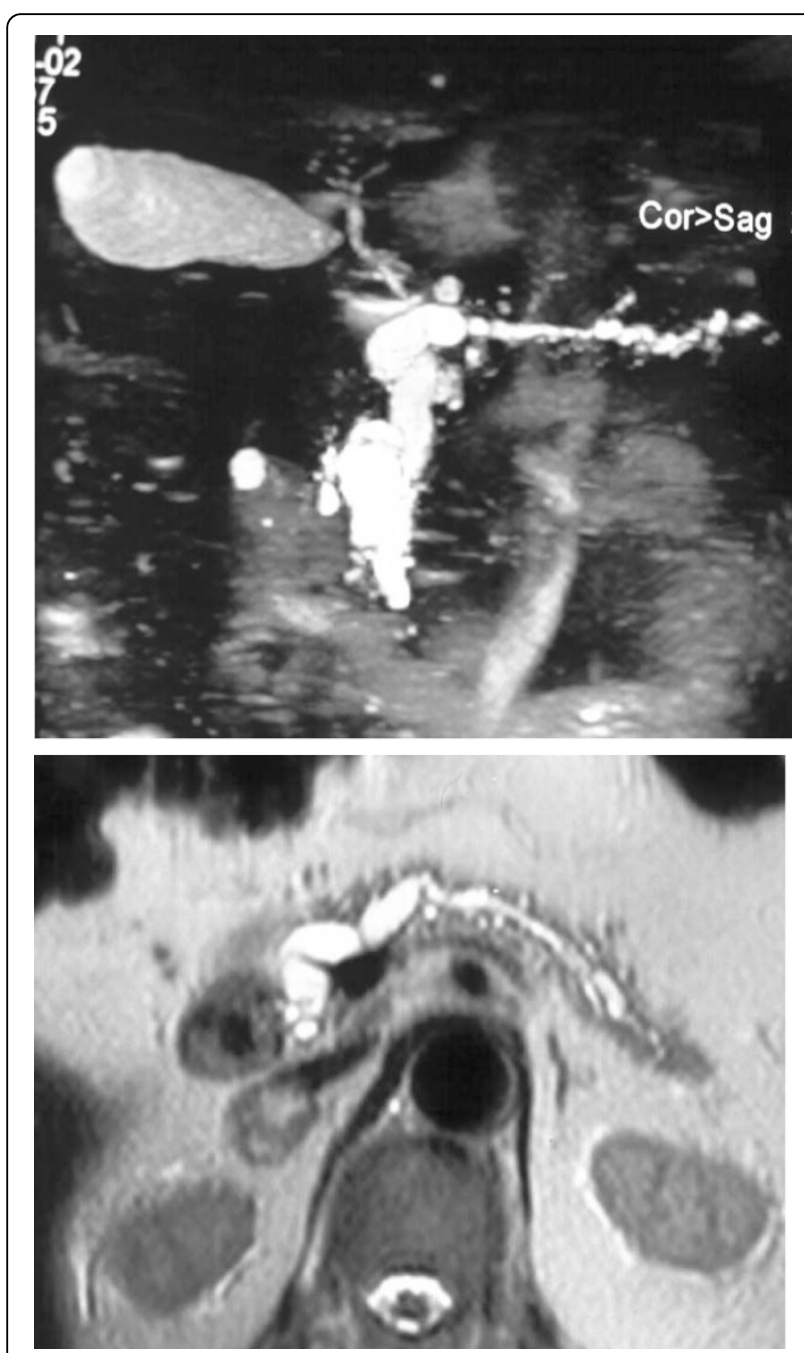

Figure 2 Colangio-MRI: main duct IPMN involving the whole gland, three-dimensional and axial reconstruction. in a 19 years period, defining a prognostic score in which the dimensional cut-off was fixed in $42 \mathrm{~mm}$ for the principal cyst and in $6.5 \mathrm{~mm}$ for the Wirsung duct, while the CA19.9 discriminant value was $35 \mathrm{U} / \mathrm{mls}$; further parameters considered were jaundice, diabetes, a pathological papilla and the principal duct involvement. Nevertheless, 19/64 patients of this series had a total score of 2 to 4 , that didn't allow to define them with certainty as benign or malignant. Among all the analyzed factors, the distinction between MDT (Fig. 2) and BDT (Fig. 3) is confirmed of maximum prognostic value in almost all the series, the second presenting a malignant potential of about 25\% (ranging from $6 \%$ to $46 \%$ ), compared to $70 \%$ for the former (60-92\%) [2]. Not all main duct IPMNs are malignant at diagnosis; nevertheless, resection should be proposed in such cases, considering that: 1) survival of the patients resected for in situ carcinoma is $100 \%$, while it is $60 \%$ in presence of infiltrative carcinoma; 2) up to now we cannot obtain by preoperative means a definitive differentiation of dysplasia from carcinoma; 3 ) the transition from a benign to a malignant form is postulated. The most representative series of main duct IPMN includes 140 patients, of which $60 \%$ presented a malignant lesion and $41 \%$ nodal metastases [5]. In our series, 10 patients have been submitted to pancreatic resection for main duct tumour: 7 had a carcinoma ( 2 in situ and 5 infiltrating), multifocal in 3 cases and 1 patient had nodal metastases.

Results are encouraging in this subset of pancreas cancer: just the patient with nodal metastases died 16 months after the intervention, while the remaining 6 are all alive and disease free, with a follow up ranging from 15 and 70 months (mean 42 months). Other

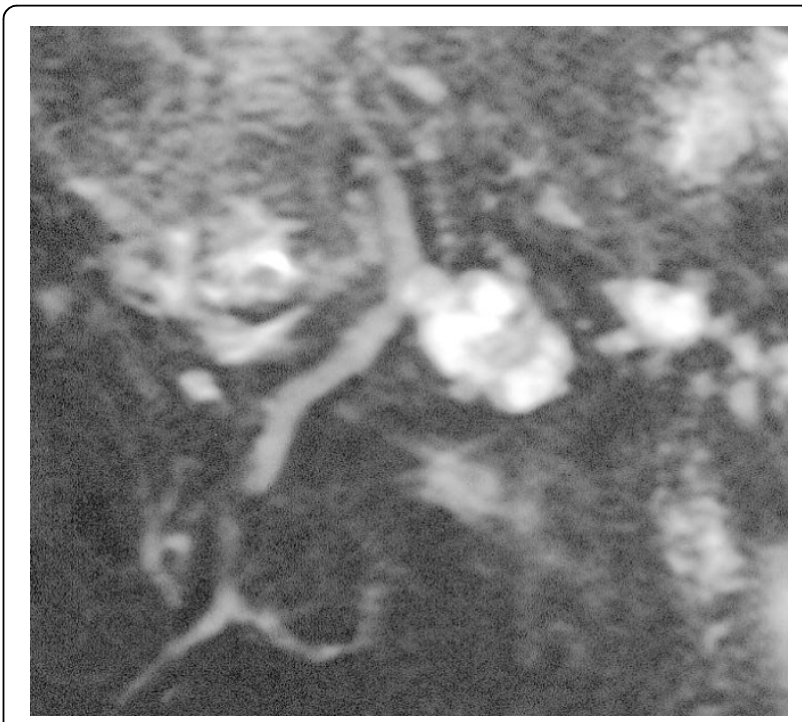

Figure 3 Colangio-MRI: branch duct IPMN, clear documentation of the communication with the Wirsung duct. 
studies also reported good outcomes for the resected patients, being 5 year disease free survival rate about $90 \%$ for patient with non invasive cancer, $80 \%$ for invasive cancers and $65 \%$ for patients with nodal metastases $[6,9,21]$. Thus, with an almost certain diagnosis of intraductal neoplasia involving the Wirsung, a demolitive surgery appears to be justified.

An at least partial conservation of the gland to preserve its exocrine and endocrine function should be part of the strategy of the operation to guarantee a good quality of life to patients who are expected to survive for a long time, and often definitely recover. Nevertheless, the recurrent multifocal involvement of the Wirsung duct sets a limit to the respect of such principle. In our series, we were obliged to extend the resection to the whole gland in 6 out of 10 cases, while in 3 we proceeded to a distal resection and in 1 to an enlarged proximal resection (subtotal duodeno-pancreatectomy). The patients submitted to total pancreatectomy had an obvious whole Wirsung disease from preoperative imaging in 4 cases, while in the remaining 2 the intraoperative frozen sections examination imposed the extension of the pancreas demolition. A recent paper reporting 127 partial pancreatectomies for IPMN shows the difficulty to perform limited resections: in $29 \%$ of the cases it was necessary to extend the resection, up to a maximum of 4 re-resections, during the same operation, to obtain a negative margin [23]. In $8 \%$ of the this series the Wirsung duct was de-epithelized at the margin and this alteration seems to represent a significant prognostic factor for recurrence; this was reported also previously [24]. In the same series the definitive diagnosis at the margin of the resection was different from the intra-operative results in $6 \%$ of the cases. In $19 \%$ of 43 patients analyzed by Eguchi through a complex strategy of separate cytology in the different lines of the gland [25], the IPMN resulted to be discontinuous along the Wirsung duct. Moreover the definition of positive section margin is not established: for some Authors it consists in the presence of intraductal papillomatosis even in absence of displasia, for others the displasia of various degree must be present [2].

The branch duct IPMNs (Fig. 3), on the other side, have a better behaviour and prognosis, similar to cystic mucinous neoplasm. In the Verona-Boston series of 145 resected patients, only $22 \%$ harboured a malignant cancer, all well characterized in their morphology by a cyst greater than $3 \mathrm{~cm}$, with a wall greater that $3 \mathrm{~mm}$, and nodules or papillae in the cyst [26]. The same Authors reported 163 patient resected for cystic mucinous tumor, $17.5 \%$ of which were malignant, but never when maximum size was lower than $4 \mathrm{~cm}$ and in absence of nodules/papillae [27]. The 26 branch duct IPMNs included in our paper, 3 resected and 23 observed, from the first observation had been classified as benign and they confirmed their benign nature at the histopathology for the resected ones or, if not operated, showing no change at a 28 months mean follow-up. This is in accord with the results of a recent publications from an American multicentric [28] and a Japanese monocentric [29] series; in both studies intraductal branch-duct tumours followed without surgery, clearly showed that it is rarely necessary to perform surgery for a dimensional evolution or for the appearance of radiological elements of suspect (11/70 and 7/82 patients respectively), and that the evolution to carcinoma is rare eventuality (1 carcinoma in situ in both series). Thus, carcinoma in an intraductal branch duct tumour is to be suspected in presence of jaundice and weight loss, or cyst greater than $30 \mathrm{~mm}$ or intramural nodules. In patients who doesn't present such characteristics just an instrumental follow-up is indicated.

\section{Conclusions}

The reported series of IPMNs from a Surgical Department confirms the published guidelines: main duct IPMNs have an high risk for malignancy and should be operated on whenever possible, while for branch duct IPMNs clinical and morphological parameters may prove useful to choose the better treatment.

\section{Author details}

${ }^{1}$ Department of Medical and Surgical Sciences, Surgical Clinic, Brescia University, P.le Spedali Civili, 1, 25123 Brescia, Italy. ${ }^{2}$ Department of Medical and Surgical Sciences, Endoscopy, Brescia University, P.le Spedali Civili, 1, 25123 Brescia, Italy. ${ }^{3}$ Department of Pathology, Brescia Civil Hospital, P.le Spedali Civili, 1, 25123 Brescia, Italy. ${ }^{4}$ Department of Radiology, Brescia Civil Hospital, P.le Spedali Civili, 1, 25123 Brescia, Italy.

\section{Authors' contributions}

GLB conceived the study; GLB, NP and SMG participated in the design of the study and drafted the manuscript; FG and MC participated in the patients follow-up; CB carried out the histological analysis; GM carried out the endoscopic examinations; LG controlled all the radiological examinations. All authors read and approved the final manuscript.

\section{Competing interests}

The authors declare that they have no competing interests.

Received: 21 December 2009 Accepted: 7 April 2010

Published: 7 April 2010

\section{References}

1. Kloppel G, Solcia E, Longnecker DS, Capella C, Sobin LH: WHO: histological typing of tumors of the exocrine pancreas. Springer, Berlin, 21996.

2. Tanaka M, Chari S, Adsay V, Fernandez-del Castillo C, Falconi M, Shimizu M, Yamaguchi K, Yamao K, Matsuno S, International Association of Pancreatology: International consensus guidelines for management of intraductal papillary mucinous neoplasms and mucinous cystic neoplasms of the pancreas. Pancreatology 2006, 6:17-32.

3. Ohashi K, Murakami Y, Maruyama M, Takekoshi T, Ohta H, Ohashi I: Four cases of mucous-secreting pancreatic cancer. Progr Digest Endosc 1982, 20:348-351.

4. Kloppel G: Clinicopathologic view of intraductal papillary- mucinous tumor of the pancreas. Hepatogastroenterology 1998, 45:1981-5. 
5. Salvia R, Fernández-del Castillo C, Bassi C, Thayer SP, Falconi M, Mantovani W, Pederzoli P, Warshaw AL: Main-duct intraductal papillary mucinous neoplasms of the pancreas: clinical predictors of malignancy and long-term survival following resection. Ann Surg 2004, 239:678-85, discussion 685-7.

6. Longnecker DS, Hruban RH, Kloppel G: Intraductal-papillary mucinous neoplasms of the pancreas. World Health Organization classification of tumors. Pathology and genetics of tumors of the digestive system Lyon: IARC pressHamilton SR, Aaltonen LA 2000, 237-240.

7. Baiocchi GL, Portolani N, Bertagna F, Gheza F, Pizzocaro C, Giubbini R, Giulini SM: Possible additional value of 18FDG-PET in managing pancreas intraductal papillary mucinous neoplasms: preliminary results. J Exp Clin Cancer Res 2008, 27:10

8. Adsay NV, Conlon KC, Zee SY, Brennan MF, Klimstra DS: Intraductal papillary-mucinous neoplasms of the pancreas: an analysis of in situ and invasive carcinomas in 28 patients. Cancer 2002, 94:62-77.

9. Cellier C, Cuillerier E, Palazzo L, Rickaert F, Flejou JF, Napoleon B, Van Gansbeke D, Bely N, Ponsot P, Partensky C, Cugnenc PH, Barbier JP, Devière J, Cremer M: Intraductal papillary and mucinous tumors of the pancreas: accuracy of preoperative computed tomography, endoscopic retrograde pancreatography and endoscopic ultrasonography, and longterm outcome in a large surgical series. Gastrointest Endosc 1998, 47:42-9.

10. Irie H, Yoshimitsu K, Aibe H, Tajima T, Nishie A, Nakayama T, Kakihara D, Honda $\mathrm{H}$ : Natural history of pancreatic intraductal papillary mucinous tumor of branch duct type: follow up study by magnetic resonance cholangiopancreatography. J Comput Assist Tomogr 2004, 28:117-22.

11. Yamao K, Ohashi K, Nakamura T, Suzuki T, Shimizu Y, Nakamura $Y$, Horibe $Y$, Yanagisawa A, Nakao A, Nimuara $Y$, Naito $Y$, Hayakawa $T$ : The prognosis of intraductal papillary mucinous tumours of the pancreas. Hepatogastroenterology 2000, 47:1129-1134.

12. Kimura W, Nagai H, Kuroda A, Muto T, Esaki Y: Analysis of small cystic lesions of the pancreas. Int J Pancreatol 1995, 18:197-206.

13. Aithal GP, Chen RY, Cunningham JT, Durkalski V, Kim EY, Patel RS, Wallace MB, Hawes RH, Hoffman BJ: Accuracy of EUS for detection of intraductal papillary mucinous tumor of the pancreas. Gastrointest Endosc 2002, 56:701-7.

14. Brugge WR: Cystic pancreatic lesions: can we diagnose them accurately what to look for? FNA marker molecular analysis resection, surveillance, or endoscopic treatment? Endoscopy 2006, 38(Suppl 1):S40-7.

15. Sperti C, Bissoli S, Pasquali C, Frison L, Liessi G, Chierichetti F, Pedrazzoli S: 18-fluorodeoxyglucose positron emission tomography enhances computed tomography diagnosis of malignant intraductal papillary mucinous neoplasms of the pancreas. Ann Surg 2007, 246:932-7.

16. Chari ST, Yadav D, Smyrk TC, DiMagno EP, Miller LJ, Raimondo M, Clain JE, Norton IA, Pearson RK, Petersen BT, Wiersema MJ, Farnell MB, Sarr MG: Study of recurrence after surgical resection of intraductal papillary mucinous neoplasm of the pancreas. Gastroenterology 2002, 123:1500-1507.

17. Doi R, Fujimoto K, Wada M, Imamura M: Surgical management of intraductal papillary mucinous tumor of the pancreas. Surgery 2002, 132:80-85.

18. Maire F, Hammel P, Terris B, Paye F, Scoazec JY, Cellier C, Barthet M O'Toole D, Rufat P, Partensky C, Cuillerier E, Lévy P, Belghiti J, Ruszniewski P: Prognosis of malignant intraductal papillary mucinous tumours of the pancreas after surgical resection. Comparison with pancreatic ductal adenocarcinoma. Gut 2002, 51:717-22.

19. Bassi C, Procacci C, Zamboni G, Scarpa A, Cavallini G, Pederzoli P: Intraductal papillary mucinous tumors of the pancreas. Int J Pancreatol 2000, 27:181-93

20. Kobari M, Egawa S, Shibuya K, Shimamura H, Sunamura M, Takeda K, Matsuno S, Furukawa T: Intraductal papillary mucinous tumors of the pancreas comprise 2 clinical subtypes: differences in clinical characteristics and surgical management. Arch Surg 1999, 134:1131-6.

21. Terris $B$, Ponsot $P$, Paye $F$, Hammel $P$, Sauvanet $A$, Molas $G$, Bernades $P$, Belghiti J, Ruszniewski $P$, Fléjou JF: Intraductal papillary mucinous tumors of the pancreas confined to secondary ducts show less aggressive pathologic features as compared with those involving the main pancreatic duct. Am J Surg Pathol 2000, 24:1372-7.

22. Fujino Y, Matsumoto I, Ueda T, Toyama H, Kuroda Y: Proposed new score predicting malignancy of intraductal papillary mucinous neoplasms of the pancreas. Am J Surg 2007, 194:304-7.
23. Couvelard A, Sauvanet A, Kianmanesh R, Hammel P, Colnot N, Lévy P, Ruszniewski $P$, Bedossa P, Belghiti J: Frozen sectioning of the pancreatic cut surface during resection of intraductal papillary mucinous neoplasms of the pancreas is useful and reliable: a prospective evaluation. Ann Surg 2005, 242:774-8, discussion 778-80.

24. Falconi M, Salvia R, Bassi C, Zamboni G, Talamini G, Pederzoli P: Clinicopathological features and treatment of intraductal papillary mucinous tumor of the pancreas. Br J Surg 2001, 88:376-381.

25. Eguchi $H$, Ishikawa $O$, Ohigashi $H$, Sasaki $Y$, Yamada T, Nakaizumi A, Uehara H, Takenaka A, Kasugai T, Imaoka S: Role of intraoperative cytology combined with histology in detecting continuous and skip type intraductal cancer existence for intraductal papillary mucinous carcinoma of the pancreas. Cancer 2006, 107:2567-75.

26. Rodriguez JR, Salvia R, Crippa S, Warshaw AL, Bassi C, Falconi M, Thayer SP, Lauwers GY, Capelli P, Mino-Kenudson M, Razo O, McGrath D, Pederzoli P, Fernández-Del Castillo C: Branch-duct intraductal papillary mucinous neoplasms: observations in 145 patients who underwent resection. Gastroenterology 2007, 133:72-9.

27. Crippa S, Salvia R, Warshaw AL, Domínguez I, Bassi C, Falconi M, Thayer SP, Zamboni G, Lauwers GY, Mino-Kenudson M, Capelli P, Pederzoli P, Castillo CF: Mucinous Cystic Neoplasm of the Pancreas is Not an Aggressive Entity: Lessons From 163 Resected Patients. Ann Surg 2008, 247:571-579.

28. Pelaez-Luna M, Chari ST, Smyrk TC, Takahashi N, Clain JE, Levy MJ, Pearson RK, Petersen BT, Topazian MD, Vege SS, Kendrick M, Farnell MB: Do consensus indications for resection in branch duct intraductal papillary mucinous neoplasm predict malignancy? A study of 147 patients. Am J Gastroenterol 2007, 102:1759-64

29. Tanno S, Nakano Y, Nishikawa T, Nakamura K, Sasajima J, Minoguchi M, Mizukami Y, Yanagawa N, Fujii T, Obara T, Okumura T, Kohgo Y: Natural history of branch duct intraductal papillary-mucinous neoplasms of the pancreas without mural nodules. Gut 2008, 57:339-43.

\section{doi:10.1186/1477-7819-8-25}

Cite this article as: Baiocchi et al:: Intraductal papillary mucinous neoplasm of the pancreas (IPMN): clinico-pathological correlations and surgical indications. World Journal of Surgical Oncology 2010 8:25.

\section{Submit your next manuscript to BioMed Central and take full advantage of:}

- Convenient online submission

- Thorough peer review

- No space constraints or color figure charges

- Immediate publication on acceptance

- Inclusion in PubMed, CAS, Scopus and Google Scholar

- Research which is freely available for redistribution

Submit your manuscript at www.biomedcentral com/submit
C Biomed Central 